\title{
Enhanced relapse prevention for bipolar disorder by community mental health teams: cluster feasibility randomised trial
}

\author{
F. Lobban, L. Taylor, C. Chandler, E. Tyler, P. Kinderman, R. Kolamunnage-Dona, C. Gamble, \\ S. Peters, E. Pontin, W. Sellwood and R. K. Morriss
}

\section{Background}

Relapse prevention for bipolar disorder increases time to relapse but is not available in routine practice.

\section{Aims \\ To determine the feasibility and effectiveness of training community mental health teams (CMHTs) to deliver enhanced relapse prevention.}

\section{Method}

In a cluster randomised controlled trial, $\mathrm{CMHT}$ workers were allocated to receive $12 \mathrm{~h}$ training in enhanced relapse prevention to offer to people with bipolar disorder or to continue giving treatment as usual. The primary outcome was time to relapse and the secondary outcome was functioning.

\section{Results}

Twenty-three CMHTs and 96 service users took part. Compared with treatment as usual, enhanced relapse prevention increased median time to the next bipolar episode by 8.5 weeks (hazard ratio $0.79,95 \% \mathrm{Cl} 0.45-1.38$ ). Social and occupational functioning improved with the intervention (regression coefficient 0.68, 95\% Cl 0.05-1.32). The clustering effect was negligible but imprecise (intracluster correlation coefficient $0.0001,95 \% \mathrm{Cl} 0.0000$ 0.5142)

\section{Conclusions}

Training care coordinators to offer enhanced relapse prevention for bipolar disorder may be a feasible effective treatment. Large-scale cluster trials are needed.

\section{Declaration of interest}

None.
Recurrence rates for mania and depression in bipolar disorder are high: around $50 \%$ at 1 year and $70 \%$ at 4 years. ${ }^{1,2}$ Relapse prevention teaches people to recognise and manage early warning signs of mania and depression, and can increase time to recurrence, reduce the need for hospitalisation and improve functioning. ${ }^{3}$ However, previous studies used specialist services, expert therapists or extensive therapy, ${ }^{4-6}$ which are not routinely available in mainstream services such as the UK National Health Service (NHS). This study examined the feasibility of a cluster randomised controlled trial (RCT) of an enhanced form of relapse prevention $v$. treatment as usual delivered by frontline care staff (care coordinators) in community mental health teams (CMHTs), and collected data to support a meaningful sample size calculation to be made for a phase III trial with a cluster RCT design. ${ }^{7}$ Hence a power calculation was not applicable.

\section{Method}

The UK Central Office of Research Ethics Committees and NHS Trust Research Offices approved this study. A detailed protocol is provided elsewhere. ${ }^{8}$ The trial is registered with the International Standard Randomised Controlled Trials Registry, number ISRCTN41352631.

\section{Trial design}

A cluster RCT was conducted across four NHS trusts, each responsible for several CMHTs. Randomisation was stratified by trust, with CMHT as the unit of randomisation. A cluster design was necessary as service users access support from different members of the CMHT, who all need training to respond appropriately. Training in relapse prevention would also influence how care coordinators worked with all service users, so offering both relapse prevention and treatment as usual within one team would not be possible without contamination between treatments.

\section{Participants}

Participants were the CMHTs, the care coordinators and service users who fulfilled the inclusion criteria. A CMHT is a multidisciplinary team of health professionals covering a specific locality. Care coordinators are rarely trained in psychotherapy for bipolar disorder and focus mainly on monitoring medication, emotional support and practical issues such as housing and finance. ${ }^{9}$

\section{Inclusion and exclusion criteria}

All CMHTs across four north-west UK NHS trusts were invited to participate. Teams were included if a minimum of four care coordinators consented to training and to offering the intervention to at least three service users. Teams working from the same building with extensive shared clinical cover were entered as a single team to reduce contamination between teams allocated to different arms of the trial. Clients of the teams were invited to participate if they had a lifetime diagnosis of bipolar disorder type 1 or 2 , had experienced two or more relapses ever, with at least one in the past year or two in the past 3 years, and had not experienced a major episode in the previous 4 weeks. They were excluded if they had a clear organic cause of their disorder, rapid cycling disorder, a primary diagnosis of alcohol or drug misuse, a poor understanding of English or were unable or unwilling to give written and oral informed consent.

\section{Procedure}

All participating teams were given a verbal presentation and written information about the study. Participants with a clinical 
diagnosis of bipolar disorder were initially informed about the study by their care coordinator and, where possible, were recruited prior to the randomisation of teams. Individuals with a clinical diagnosis of bipolar disorder were assessed by researchers (C.C., E.T.) masked to allocation, using an interview to confirm diagnosis, ${ }^{10}$ and a range of measures at baseline and at 12 weeks, 24 weeks, 36 weeks and 48 weeks following the onset of treatment. Participants receiving enhanced relapse prevention were invited to involve a relative, friend or carer. All participants gave written consent.

\section{Intervention}

Key elements of enhanced relapse prevention were those explicitly recommended by the National Institute for Health and Clinical Excellence (NICE) guidelines for bipolar disorder: psychoeducation, detailed analysis of previous episodes, identification of triggers and early warning signs, enhancing coping strategies for mood changes, action plans and an agreement with clinical services about responses to different stages of relapse. ${ }^{11}$ Elements are developed separately for mania, depression and mixed episodes. Enhanced relapse prevention is delivered alongside other interventions and builds on earlier approaches, ${ }^{12}$ but has increased focus on identifying early warning signs of depression, more detailed development of coping strategies, and involvement of a relative or friend. ${ }^{7}$ It is delivered over six $1 \mathrm{~h}$ sessions. A manual detailing the rationale and process forms the basis of a collaborative process between care coordinators and service users.

Care coordinators were trained over six $2 \mathrm{~h}$ weekly sessions by an experienced nurse (L.T.) and training followed the six-session format of the intervention. The aim of each session was to ensure that care coordinators understood the rationale and content of the corresponding therapy session, and were able to work with participants to achieve the session aims. Training methods included presentations, videotaped examples (using professional actors), group discussion and role-play exercises. Following training, care coordinators were offered weekly supervision for 3 months during which time they were expected to offer the intervention to at least one participating service user. Group supervision was offered to care coordinators within the same team and was conducted by the trial therapist (L.T.). Feedback on training and supervision was very positive. ${ }^{13}$

\section{Measures}

The primary outcome for estimating the effect size of the intervention was time to recurrence of any episode of major depression, hypomania, mania or mixed affective state satisfying DSM-IV criteria, ${ }^{14}$ using a measure ${ }^{15}$ which combines questions on the Structured Clinical Interview for DSM-IV (SCID), ${ }^{10}$ the Hamilton Rating Scale for Depression, the Beck Rafaelsen Mania Rating Scale and the Longitudinal Interview Follow-up Evaluation (LIFE-II) following a period of at least 8 weeks below episodic threshold for that pole. For the 15 participants for whom interviews were unfeasible, relapse was identified from clinical notes and defined as a clear documented description of change in mental state towards mania or depression in combination with a documented change in treatment or management. ${ }^{16}$ Secondary outcome was functioning, assessed using the Social and Occupational Functioning Assessment Scale (SOFAS). ${ }^{17}$

\section{Quality assurance}

Two researchers were trained on all the measures. Interrater reliability was assessed at baseline for 16 participants. To preserve the masking of the researchers, care coordinators and participants were reminded at the outset of each encounter not to say anything that might indicate which treatment arm they were in. No clinical notes were screened between randomisation and prior to completion of assessments. Any instances of unmasking (failure of masking) were recorded.

\section{Statistical analysis}

Analyses were conducted on an intention-to-treat basis. Statistical significance was set at $P<0.05$ and analyses were conducted in the statistical package R version 2.9.2 for Windows, ${ }^{18}$ adjusted for the cluster design of the study. The time to recurrence was analysed using Kaplan-Meier curves. The significance tests were based on the shared frailty model, an extension of the Cox proportional hazards regression model. Separate analyses were conducted for three different types of recurrence (any, depressive and hypomanic/manic episodes), using the SURVIVAL package in R. The level of dependence or heterogeneity among patterns of recurrence within the same team was estimated by the intracluster correlation coefficient (ICC) using estimate of frailty from the shared frailty model. ${ }^{19}$ An approximate $95 \%$ bootstrap-based confidence interval for ICC was computed by the 2.5 and 97.5 percentiles of the ordered list of bootstrap replications of ICC.

Analyses of SOFAS scores were conducted by means of linear mixed-effects regression models using the NLME package in $\mathrm{R}$ language, using maximum likelihood to estimate regression parameters. In unadjusted analyses treatment effect was included as the only covariate. In adjusted analyses, treatment effects along with variables considered of clinical importance and variables with imbalance at baseline were considered for inclusion in the model.

\section{Results}

The flow of teams and individuals through the study is shown in Fig. 1. The size of the teams was similar in both arms: the median number of full-time staff was 11 (range 5.5-35) for the intervention and 11 (range 6-20) for treatment as usual. There was no difference between the demographic and clinical characteristics of the participants in each arm, except that more participants in the usual treatment arm had children (online Table DS1).

\section{Reliability and quality assurance}

Interrater reliability between the researchers was excellent, with $100 \%$ agreement on diagnostic categorisation using the SCID and on history of bipolar episodes. Only 4\% (5 of 128 pairs) of scores on weekly mood ratings over 4 weeks differed and all were within one point of each other. Both researchers reported total 'unmasking. Because of the cluster design, unmasking for one participant resulted in unmasking for the CMHT and all their trial participants. The most common reason was from contact with care coordinators prior to follow-up interviews in order to conduct risk assessments. Attempts to recruit participants prior to randomisation also failed and approximately half of participants were recruited after randomisation. Exact figures are not possible because some people recruited by care coordinators prior to randomisation were subsequently found to be ineligible, failed to consent to the study or consented after randomisation.

\section{Recurrence}

Of the 96 service users - 40 in the treatment as usual (TAU) group and 56 in the enhanced relapse prevention (ERP) group - over 


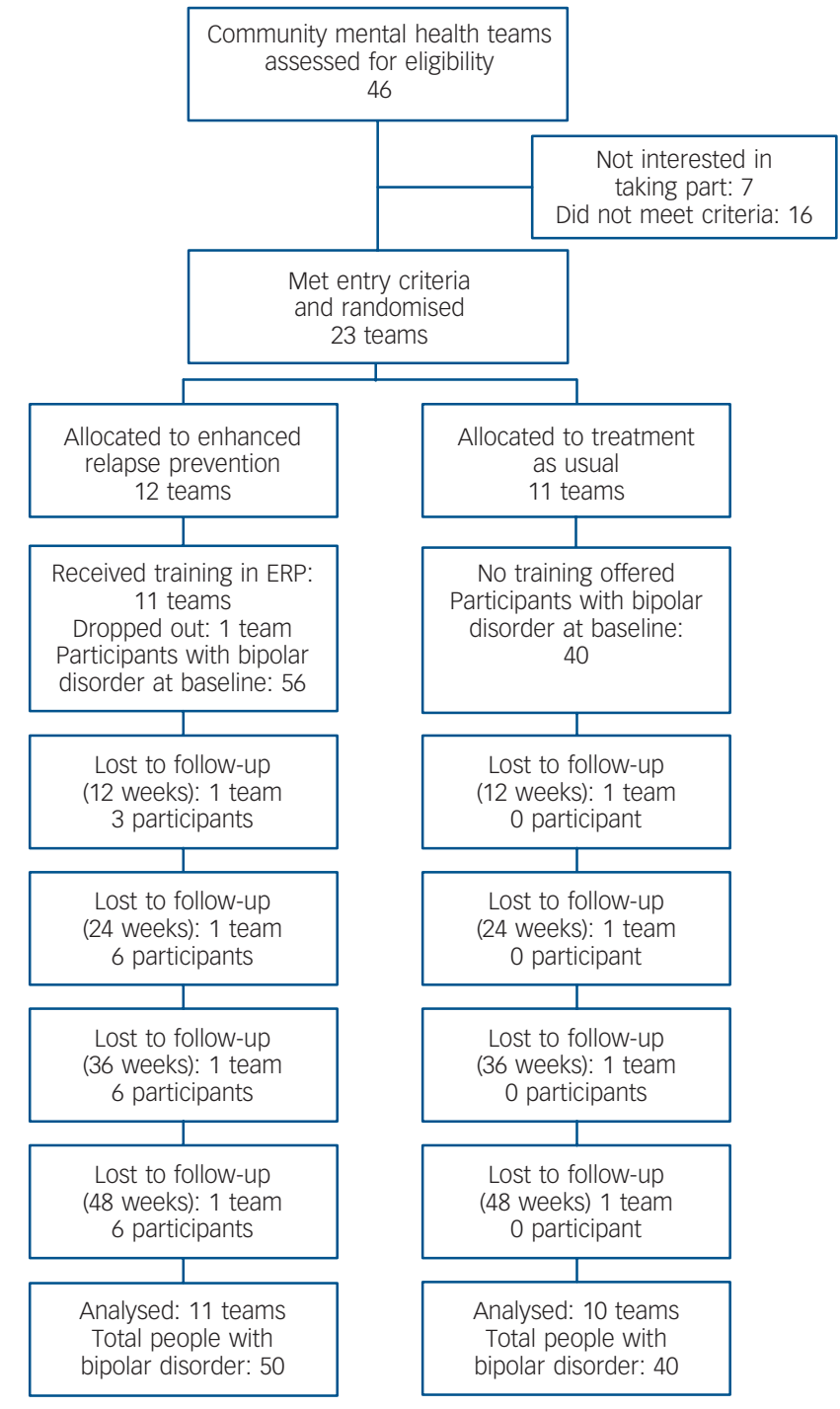

Fig. 1 Flow of teams and clients through the study.

half $(52 \%, n=50)$ had a recurrence (depressive or manic) during follow-up: $60 \%(n=24)$ of participants receiving usual treatment and $46 \%(n=26)$ of those receiving the intervention. Of the first recurrences $66 \%(n=33)$ were depressive episodes (TAU $67 \%$, $n=16$; ERP $65 \%, n=17)$ and $34 \%(n=17)$ were manic or hypomanic (TAU 33\%, $n=8$; ERP 35\%, $n=9$ ). The ICC was negligible, at 0.0001 (95\% CI $0.0000-0.5142)$.

Figure 2 shows the Kaplan-Meier estimates for any recurrence. The numbers of participants recurrence-free and still in follow-up (numbers at risk) and number of cumulative events in each treatment group are given for each assessment point. The estimated median survival time to any recurrence was 42 weeks for the ERP group and 33.5 weeks for the TAU group (half of the participants offered relapse prevention stayed free from episodes for 8.5 weeks longer than those receiving usual treatment). The unadjusted hazard ratios of time to first bipolar episode, time to first depressive episode and time to first manic episode were 0.79 (95\% CI 0.45-1.38), 0.78 (95\% CI 0.39-1.53) and $0.82(95 \%$ CI $0.32-2.13)$ respectively. These results show a non-significant reduction favouring the intervention. Number of previous episodes $(P<0.0001)$ and education $(P=0.0003)$ were associated with time to first bipolar episode, giving an adjusted hazard ratio of time to first bipolar episode of 0.62 (95\% CI

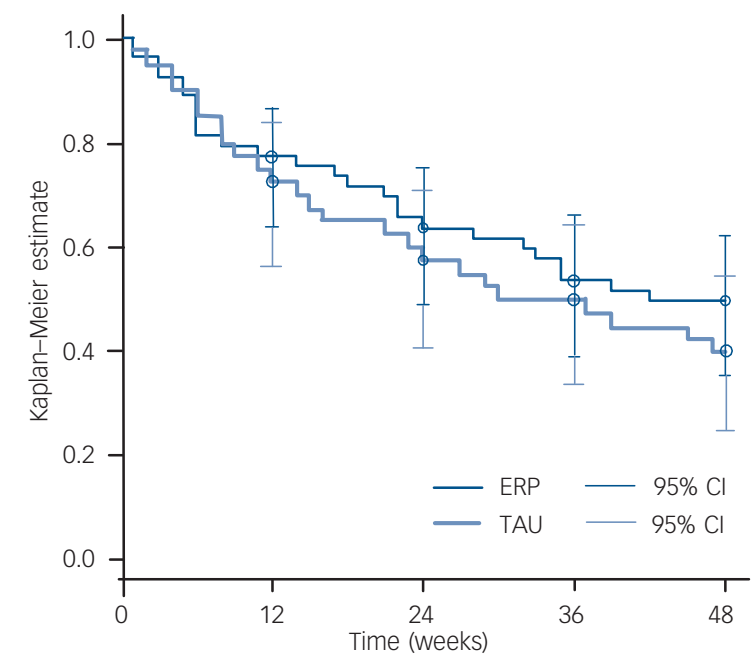

Number at risk (Number of events)

$\begin{array}{llllll}\text { ERP } & 56(0) & 41(12) & 33(18) & 27(24) & 25(26) \\ \text { TAU } & 40(0) & 30(10) & 24(16) & 20(20) & 16(24)\end{array}$

Fig. 2 Kaplan-Meier estimates of time to first bipolar episode for the groups receiving enhanced relapse prevention (ERP) or treatment as usual (TAU), with 95\% confidence intervals.

0.34-1.15). Number of previous episodes $(P=0.0103)$ was associated with time to first depressive episode, giving an adjusted hazard ratio of time to first depressive episode of 0.74 (95\% CI $0.37-1.48$ ). There were too few events to calculate an adjusted hazard ratio for time to relapse in mania.

\section{Social and occupational functioning}

Scores on the SOFAS were available for 87 participants at baseline, 73 at 12 weeks, 69 at 24 weeks and 70 at 48 weeks. Both education and number of previous episodes were included in the model. There was no overall effect of time (regression coefficient $-0.43,95 \%$ CI -0.91 to 0.05$)$ or treatment arm $(3.23,95 \%$ CI -2.83 to 9.29 ), but there was a significant time $\times$ treatment interaction $(0.68,95 \%$ CI 0.05 to 1.32$)$ suggesting a significant difference in pattern of change over time between the groups, favouring the intervention.

\section{Discussion}

This is the first study to demonstrate that training care coordinators to offer enhanced relapse prevention is feasible and may improve outcome for people with bipolar disorder. As the intervention is brief and does not require highly trained therapists, it has the potential to be made widely available using experienced mental health professionals who are already in routine contact with people with bipolar disorder.

\section{Main findings}

The primary outcome variable - time to recurrence of an episode of depression or mania/hypomania - was increased in people receiving enhanced relapse prevention compared with those receiving treatment as usual by an average of almost 9 weeks. The risk of having an episode during the follow-up period was $21 \%$ and $38 \%$ lower for participants receiving the intervention than for those receiving usual treatment in the unadjusted and adjusted analyses respectively. Although comparable in effect size 
to other studies of relapse prevention approaches, ${ }^{5}$ the effect of the intervention on time to first bipolar recurrence was not significant. However, this is a feasibility study and was not powered to detect such differences. All analyses produced large confidence intervals, suggesting that caution and further research are necessary. Like some but not all previous RCTs of psychological treatments for bipolar disorder, ${ }^{20}$ and also naturalistic studies of bipolar disorder, ${ }^{21}$ number of previous bipolar episodes was associated with a worse outcome for participants in both groups. We found that education influenced outcome, but other studies have not found a consistent effect of this or other demographic variables on outcome. ${ }^{22}$

There was a statistically significant difference between the two study arms in social and occupational functioning. Functioning increased over time in the relapse prevention group relative to usual treatment, a finding consistent with other relapse prevention interventions, where such effects have also emerged over time and are as large as their effects on symptoms. ${ }^{4,12,22}$

This trial was also designed to assess the effect of the cluster design. Although the point estimate of ICC was small, there is considerable imprecision which is reflected by the large upper limit on the 95\% confidence interval. The impact of the clustering effect should consider the full range of values in the interval and therefore it is difficult to justify a negligible clustering effect in further large-scale trials.

\section{Study limitations}

The sample size and number of relapses during the follow-up period were both small and therefore the confidence intervals are large, making it difficult to draw firm conclusions. However, as the study aimed to determine whether it would be possible to conduct a larger phase III trial of the same design, ${ }^{7}$ definitive conclusions were not the primary objective. The overall rate of relapse in this study was similar to that in previous trials (50$60 \%$ over 1 year), ${ }^{3,5,21}$ but relatively few manic episodes occurred. The reason for this is unclear since at baseline high lifetime rates of both depressive and manic relapse were reported. Strengths of the study were low loss to follow-up and the reliability of the assessment of mental state.

The sample predominantly had diagnoses of type 1 bipolar disorder and comprised White and English-speaking participants, so the results may not apply to people with type 2 disorder and to other ethnic groups. The sample contained a high proportion of Axis I comorbidity, but the results may not apply to participants with comorbid personality disorder and substance use disorders, which were uncommon in this sample.

As this was a feasibility study, additional limitations were inevitable. Ideally, participants would have been followed up for longer; previous studies have shown maximum benefit of psychoeducation interventions after 2 or more years. ${ }^{4,22,23}$ A further methodological refinement would be to recruit service users before randomisation of the CMHTs to enhanced relapse prevention or treatment as usual, but there were not the resources to achieve the oversampling that is required, since many service users would have relapsed into an acute bipolar episode leading to their exclusion from the study by the time randomisation occurred. Recruitment of many participants after the CMHTs were randomised raises the possibility of selection bias (the clinical team selecting service users knowing they would be allocated to relapse prevention or usual treatment), therefore it was important in the analysis to control for any baseline demographic differences that arose. Attempts to maintain masked assessment failed. The source of this failure was liaison with care coordinators to determine whether the participants were at risk of harm to themselves or others before visits from the research assistants. Future trials would require funding to include a trial coordinator to liaise with clinical staff and participants in arranging appointments and risk assessments because the unmasking of one participant reveals the allocation of all participants within that cluster.

\section{Clinical implications}

The essential components of an effective psychological therapy for bipolar disorder remain uncertain, but this study extends the existing evidence that teaching service users to recognise and then initiate management for the early warning signs is a clinically useful intervention.,12 Importantly, it demonstrates that approaches using early warning signs can be employed by existing staff who are already regularly following up these people, and do not necessarily require extensive training in psychological therapies.

\section{Further research}

The huge public health benefits of a simple relapse prevention training package delivered by trained mental health professionals that could be offered to large numbers of people with bipolar disorder at relatively little cost justifies further research in this area. The methodological limitations of this study have been highlighted with the aim of informing a phase III trial to address these. For many people, functioning is a more important indicator of outcome than symptoms, ${ }^{24}$ and thus needs to be a focus in future work.

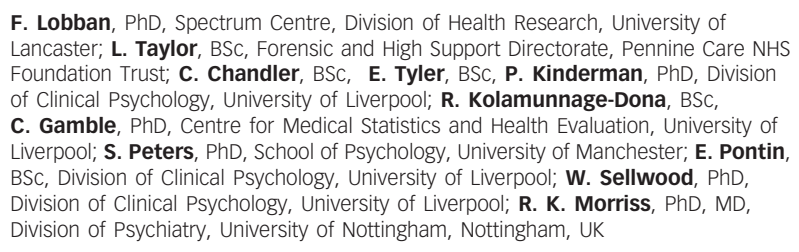

F. Lobban, PhD, Spectrum Centre, Division of Health Research, University of Lancaster; L. Taylor, BSC, Forensic and High Support Directorate, Pennine Care NHS Foundation Trust; C. Chandler, BSC, E. Tyler, BSC, P. Kinderman, PhD, Division of Clinical Psychology, University of Liverpool; R. Kolamunnage-Dona, BSC, C. Gamble, PhD, Centre for Medical Statistics and Health Evaluation, University of Liverpool; S. Peters, PhD, School of Psychology, University of Manchester; E. Pontin BSC, Division of Clinical Psychology, University of Liverpool; W. Sellwood, PhD, Division of Clinical Psychology, University of Liverpool; R. K. Morriss, PhD, MD, Division of Psychiatry, University of Nottingham, Nottingham, UK

Correspondence: Dr Fiona Lobban, Spectrum Centre, Lancaster University, Lancaster LA2 4YT, UK. Email: f.Iobban@lancaster.ac.uk

First received 26 Feb 2009, final revision 22 Jul 2009, accepted 2 Aug 2009

\section{Funding}

Funding for the work was gratefully received from the Medical Research Council and Mersey Care National Health Service Trust.

\section{References}

1 Gitlin MJ, Swendsen J, Heller TL, Hammen C. Relapse and impairment in bipolar disorder. Am J Psychiatry 1995; 152: 1635-40.

2 Altshuler LL, Gitlin MJ, Mintz J, Leight KL, Frye MA. Subsyndromal depression is associated with functional impairment in patients with bipolar disorder. J Clin Psychiatry 2002; 63: 807-11.

3 Morriss R, Mohammed F, Bolton C, McCarthy J, Williamson P, Jones A. Interventions for helping people recognise early signs of recurrence in bipolar disorder: full review. Cochrane Database Syst Rev 2007; 1: CD004854.

4 Bauer MS, McBride L, Williford WO, Glick H, Kinosian B, Altshuler L, et al. Collaborative care for bipolar disorder: Part II. Impact on clinical outcome, function, and costs. Psychiatr Serv 2006; 57: 937-45.

5 Colom F, Vieta E, Martinez-Aran A, Reinares M, Goikolea JM, Benabarre A, et al. A randomized trial on the efficacy of group psychoeducation in the prophylaxis of recurrences in bipolar patients whose disease is in remission. Arch Gen Psychiatry 2003; 60: 402-7.

6 Lam DH, Hayward P, Watkins ER, Wright K, Sham P. Relapse prevention in patients with bipolar disorder: cognitive therapy outcome after 2 years. Am J Psychiatry 2005; 162: 324-9. 
7 Medical Research Council. Developing and Evaluating Complex Interventions: New Guidance. MRC, 2008 (www.mrc.ac.uk/complexinterventionsguidance).

8 Lobban F, Gamble C, Kinderman P, Taylor L, Chandler C, Tyler E, et al. Enhanced relapse prevention for bipolar disorder - ERP trial. A cluste randomised controlled trial to assess the feasibility of training care coordinators to offer enhanced relapse prevention for bipolar disorder. BMC Psychiatry 2007; 7: 6.

9 Pontin E, Peters S, Lobban F, Rogers A, Morris RK. Enhanced relapse prevention for bipolar disorder: a qualitative investigation of value perceived for service users and care coordinators. Implementation Science 2009; 4.

10 First MB, Gibbon M, Spitzer RL, Williams JBW, Benjamin LS. Structured Clinical Interview for DSM-IV Axis I Disorders, Research Version, Patient Edition. American Psychiatric Press, 1997.

11 National Institute for Health and Clinical Excellence. Bipolar Disorder: The Management of Bipolar Disorder in Adults, Children and Adolescents in Primary and Secondary Care. NICE, 2006.

12 Perry A, Tarrier N, Morriss R, McCarthy E, Limb K. Randomised controlled trial of efficacy of teaching patients with bipolar disorder to identify early symptoms of relapse and obtain treatment. BMJ 1999; 318: 149-53.

13 Lobban F, Taylor L, Chandler C, Sellwood W, Gamble C, Tyler E, et al. Training staff in enhanced relapse prevention for bipolar disorder: uptake, skill and confidence. Psychiatr Serv 2009; 60: 702-6.

14 American Psychiatric Association. Diagnostic and Statistical Manual of Mental Disorders (4th edn) (DSM-IV). APA, 1994.

15 Paykel ES, Abbott R, Morriss R, Hayhurst H, Scott J. Sub-syndromal and syndromal symptoms in the longitudinal course of bipolar disorder. $\mathrm{Br} J$ Psychiatry 2006; 189: 118-23.
16 Rendell JM, Juszczak E, Hainsworth J, Gucht EV, Healey C, Morriss R, et al. Developing the BALANCE trial: the role of the pilot study and start-up phase. Bipolar Disord 2004; 6: 26-31.

17 Goldman $\mathrm{HH}$, Skodol AE, Lave TR. Revising axis $\mathrm{V}$ for DSM-IV: a review of measures of social functioning. Am J Psychiatry 1992; 149: 1148-56.

18 R Development Team. R Statistical Package, Version 2.9 .2 (http://www. r-project.org)

19 Lam K, Ip D. REML and ML estimation for clustered grouped survival data. Stat Med 2003; 22: 2025-34.

20 Scott J, Colom F, Vieta E. A meta-analysis of relapse rates with adjunctive psychological therapies compared to usual psychiatric treatment for bipolar disorders. Int J Neuropsychopharmacol 2007; 10: 123-9.

21 Altman S, Haeri S, Cohen L, Ten A, Barron E, Galynker II, et al. Predictors of relapse in bipolar disorder: a review. J Psychiatr Pract 2006; 126: 269-82.

22 Simon GE, Ludman EJ, Bauer MS, Unutzer J, Operskalski B. Long-term effectiveness and cost of a systematic care program for bipolar disorder Arch Gen Psychiatry 2006; 63: 500-8.

23 Colom F, Vieta $E$, Sanchez-Moreno J, Palomino-Otiniano R, Reinares $M$ Goikolea J, et al. Group psychoeducation for stabilised bipolar disorders: 5-year outcome of a randomised clinical trial. Br J Psychiatry 2009; 194 $260-5$.

24 Michalak E, Yathom N, Kolesar S, Lam R. Bipolar disorder and quality of life: a patient centred perspective. Qual Life Res 2006; 15: 25-37.

\section{A marvellous recovery}

\section{Thomas Bewley}

In 1955 I was a registrar at Claybury Hospital when a woman aged 52 was transferred from a general hospital, where she had been for several weeks following a stroke after which there were no signs of recovery. She could not speak, did not appear to be aware of her surroundings and needed spoon-feeding and total nursing care. She was therefore admitted directly to a long-stay psychogeriatric ward, without having been seen by a consultant in the normal admission ward. When I examined her I found she was in better physical health than I might have expected and she was free of any signs of pressure sores. Two days after admission she moved her limbs slightly and said 'Thank you' to a nurse who was feeding her. On each of the following days there was a steady improvement in her condition. The nurses on this ward, who were not accustomed to seeing such improvements, were surprised by the changes. I encouraged them to help her to sit up, stand up, move to a chair, walk a little with help, walk further without help, until she gradually became able to look after herself. Her speech improved at the same time, an occasional slurred word was followed by clearer sentences and within 3 weeks she was talking normally. She had no idea of what had led to her being in hospital, but accepted the fact that she had been seriously ill. Her family, who visited her daily, were very impressed and delighted by her rapid return to her old self. Five weeks after admission she had few obvious sequelae and went home. Her family thanked me very warmly for my endeavours. They told me that the very best thing that had ever happened to their mother was her transfer from a hospital where nothing could be done to a mental hospital where modern psychiatric treatment brought about cure. I told them that I had done little but that the nurses had been very good in helping to rehabilitate her. 'No, no,' they said 'if it wasn't for you, Dr Bewley, none of this would have happened.' I think they saw me as the greatest doctor since Paracelsus. In my next 40 years as a psychiatrist I never came across a more grateful family. 